\title{
Pion-delta sigma-term
}

\author{
I. P. Cavalcante, ${ }^{1, *}$ M. R. Robilotta $,{ }^{2}, \dagger$ J. Sá Borges,${ }^{3, \ddagger}$ \\ D. de O. Santos, ${ }^{1}$ and G. R. S. Zarnauskas ${ }^{2, \S}$ \\ ${ }^{1}$ Depto. de Física, CCET, Universidade Federal de Mato Grosso do Sul, \\ C.P. 549, C.E.P. 79070-900, Campo Grande, MS, Brazil. \\ ${ }^{2}$ Instituto de Física, Universidade de São Paulo, \\ C.P. 66318, 05315-970, São Paulo, SP, Brazil. \\ ${ }^{3}$ Universidade do Estado do Rio de Janeiro, Instituto de Física, \\ Rua São Francisco Xavier, 524, Maracanã, Rio de Janeiro, RJ, Brazil.
}

\begin{abstract}
We use a configuration space chiral model in order to evaluate nucleon and delta $\sigma$-terms. Analytic expressions are consistent with chiral counting rules and give rise to expected non-analytic terms in the chiral limit. We obtain the results $\sigma_{N}=46 \mathrm{MeV}$ and $\sigma_{\Delta}=32 \mathrm{MeV}$, which are very close to values extracted from experiment and produced by other groups.
\end{abstract}

PACS numbers: 13.75.Gx, 11.30.Rd

\footnotetext{
*ipcavalcante@dfi.ufms.br

${ }^{\dagger}$ robilotta@if.usp.br

${ }^{\ddagger}$ saborges@uerj . br

§gabrielz@if.usp.br
} 


\section{INTRODUCTION}

The delta $(\Delta)$ plays a very important role in low-energy pion-nucleon $(\pi N)$ scattering and correlated processes, such as the nucleon-nucleon interaction. Its contribution as an intermediate state, in many instances, supersedes that of the the nucleon. This happens for two main reasons. The first one is that the $\pi N \Delta$ coupling constant is rather large, whereas the other is related to chiral symmetry.

At low energies, pion-hadron interactions are well described by effective theories, in which an approximate $S U(2) \times S U(2)$ symmetry, broken by the pion mass $(\mu)$, accounts for the smallness of the $u$ and $d$ quark masses. In this framework, elastic pion-baryon scattering is dominated by diagrams involving both contact terms and propagating states. In order to comply with threshold chiral theorems, the latter are typically given by polynomials in small quantities, such as the pion mass or three-momenta, divided by energy denominators. When the delta is present, the scale of some denominators is given by the quantity $\omega_{\Delta}=$ $\left(M^{2}-m^{2}-\mu^{2}\right) / 2 m$, where $M$ and $m$ are respectively the delta and nucleon masses. As the difference $\Delta \equiv M-m$ is small, one has $\omega_{\Delta} \sim \Delta$. Delta contributions are given by ratios of small quantities and may turn out to be large. In such cases, numerical values adopted for $\Delta$ do influence predictions produced by effective theories, especially those that rely on the small scale expansion[1] or the heavy baryon approximation[2].

In chiral perturbation theory, there is a clear conceptual distinction between the bare baryon masses, present in the lagrangian, and their observed values, which include loop corrections. The former should, in principle, be preferred as inputs in the evaluation of theoretical amplitudes. Nevertheless, as there is little knowledge available concerning the bare delta mass, one tends to use physical values in calculations. In most cases, it is reasonable to expect that this would have little numerical importance. On the other hand, in the case of the parameter $\Delta$, which is a small quantity, the influence of loops may become relatively large.

Recently Bernard, Hemmert and Meissner[3], have stressed that the value of $\Delta_{0}$, the delta-nucleon mass splitting in the chiral limit, is an important constraint to lattice data extrapolation. The purpose of the present work is to estimate the delta $\sigma$-term, which controls the change induced in $\Delta$ when one goes from bare to physical masses. This $\sigma$ term was studied in the framework of a quark model by Lyubovitskij, Gutsche, Faessler and 
Drukarev[4] and the reader is referred to their paper for a clear formulation of the problem and earlier works.

According to the Feynman-Hellmann theorem[5] the mass $m_{B}$ of a baryon $B$ is related to its sigma-term $\sigma_{B}$ by $\sigma_{B}=\mu^{2} d m_{B} / d \mu^{2}$. Therefore the sigma-term provides a measure of the shift in the baryon mass due to chiral symmetry breaking. Whenever it is possible to evaluate $\sigma_{B}$ as a function of $\mu$, the bare mass $m_{B_{0}}$ can be extracted from the relation

$$
m_{B}=m_{B_{0}}+\int_{0}^{\mu^{2}} d \lambda \sigma_{B}(\lambda) / \lambda .
$$

As the leading term in $\sigma_{B}$ is proportional to $\mu^{2}$, it enters directly the mass shift and the difference $m_{B}-\sigma_{B}$ already provides a crude estimate for the bare value. In the case of the nucleon, one has $\sigma_{N}=45 \mathrm{MeV}[6]$, which amounts to $5 \%$ of its physical mass. In chiral perturbation theory, the leading contribution to $\sigma_{N}$ cannot be predicted theoretically. Formally, it is associated with the constant $c_{1}$ of the second order lagrangian[7, 8], which can be extracted from empirical subthreshold information. The situation of the delta is much worse, for $\pi \Delta$ scattering data are not available. One is then forced to resort to models in order to calculate the delta $\sigma$-term, which is associated with the parameter $a_{1}$ defined in ref.[1].

In this work we estimate $\sigma_{\Delta}$ using a model which proved to be successful in the case of the nucleon. Our paper is organized as follows. In section II we review our calculational procedure in the case of the nucleon and present results for the delta in section III, leaving technical details to the appendices. The main expressions for both the nucleon and delta $\sigma$-terms in configuration space are given in appendix B, written in terms of the loop integrals defined in appendix A. The consistency of our results with standard chiral counting rules is discussed in appendix $\mathrm{C}$ whereas their behaviour in the chiral limit is given in appendix D. A summary is provided in section IV.

\section{MODEL FOR THE SIGMA TERM}

In order to evaluate $\sigma_{\Delta}$, we follow a procedure used previously in the study of $\sigma_{N}(t)$, the nucleon scalar form factor[9], which is briefly reviewed here. The leading contributions to this function is $\mathcal{O}\left(q^{2}\right)$ whereas the triangle diagram, involving only known masses and coupling

constants, gives rise to corrections which begin at $\mathcal{O}\left(q^{3}\right)$ and are completely determined. At 
$\mathcal{O}\left(q^{4}\right)$, on the other hand, interactions incorporate the low energy constants $c_{1}, c_{2}$ and $c_{3}$. Data on $\pi N$ subthreshold coefficients indicate that $c_{2}$ and $c_{3}$ are larger than $c_{1}$ and that their values are approximately saturated by $\Delta$ intermendiate states[8]. Thus, up to $\mathcal{O}\left(q^{4}\right)$, the function $\sigma_{N}(t)$ can be well represented by the leading tree contribution associated with $c_{1}$, supplemented by the two triangle diagrams shown in fig.1, involving $N$ and $\Delta$ intermediate states. In the sequence we will make use of the fact that, in configuration space, contact and loop contributions split apart, since the Fourier transform acts as a filter[10]. As a result, the theoretically undetermined leading tree term yields a zero-range $\delta$-function, whereas the triangle diagrams give rise to spatially distributed structures, fully determined by known parameters.

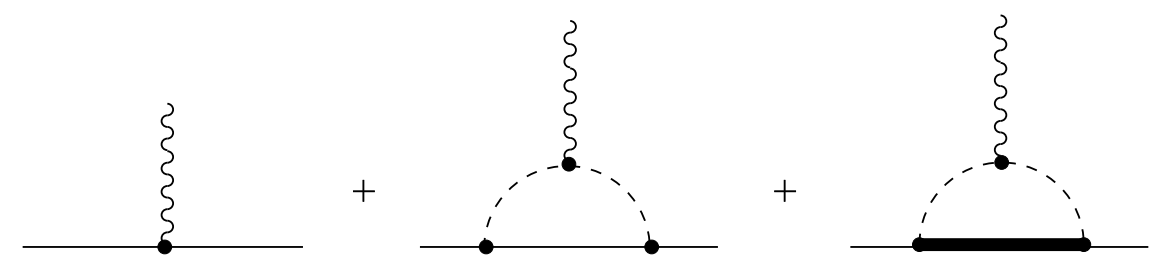

FIG. 1: Contact term and triangle diagrams contributing to the $\sigma$-term.

The nucleon scalar form factor in momentum space is defined by

$$
\left\langle N\left(p^{\prime}\right)\left|-\mathcal{L}_{s b}\right| N(p)\right\rangle=\sigma_{N}(t) \bar{u}\left(p^{\prime}\right) u(p),
$$

where $\mathcal{L}_{s b}$ is the symmetry breaking term in the lagrangian and $t=\left(p^{\prime}-p\right)^{2}$. In terms of the quark degrees of freedom, one has $\mathcal{L}_{s b}=-\hat{m}(\bar{u} u+\bar{d} d)$, with $\hat{m}=\left(m_{u}+m_{d}\right) / 2$. The configuration space scalar form factor is denoted by $\tilde{\sigma}_{N}$ and given by

$$
\tilde{\sigma}_{N}(\boldsymbol{r})=\int \frac{d^{3} q}{(2 \pi)^{3}} e^{-i \boldsymbol{q} \cdot \boldsymbol{r}} \sigma_{N}(t)
$$

with $\boldsymbol{q}=\left(\boldsymbol{p}^{\prime}-\boldsymbol{p}\right)$, in the Breit frame. The nucleon $\sigma$-term, defined as $\sigma_{N} \equiv \sigma_{N}(t=0)$, is given by

$$
\sigma_{N}=4 \pi \int_{0}^{\infty} d r r^{2} \tilde{\sigma}_{N}(r)
$$

The contributions from the diagrams of fig.1 to $\tilde{\sigma}_{N}(\boldsymbol{r})$ read

$$
\tilde{\sigma}_{N}(\boldsymbol{r})=-4 c_{1} \mu^{2} \delta^{3}(\boldsymbol{r})+\tilde{\sigma}_{N_{N}}(r)+\tilde{\sigma}_{N_{\Delta}}(r)
$$


where $\tilde{\sigma}_{N_{N}}(r)$ and $\tilde{\sigma}_{N_{\Delta}}(r)$ are given by eqs.(B4) and (B5) of appendix B and displayed in fig.2. These functions are based on unregularized loop integrals and diverge for small values of $r$. In momentum space, regularization is achieved by means of counterterms, which give rise to polynomials in $t$, designed to cancel the divergences of the loop integrals. In configuration space, this regularization procedure amounts to adding $\delta$-functions and their derivatives to $\tilde{\sigma}_{N}(r)$. This gives rise to a regularized form factor which is very large both at $r=0$ and in a sizeable vicinity of that point. We argue, in the sequence, that this picture is not consistent with the definition of the form factor given by eq.(2).

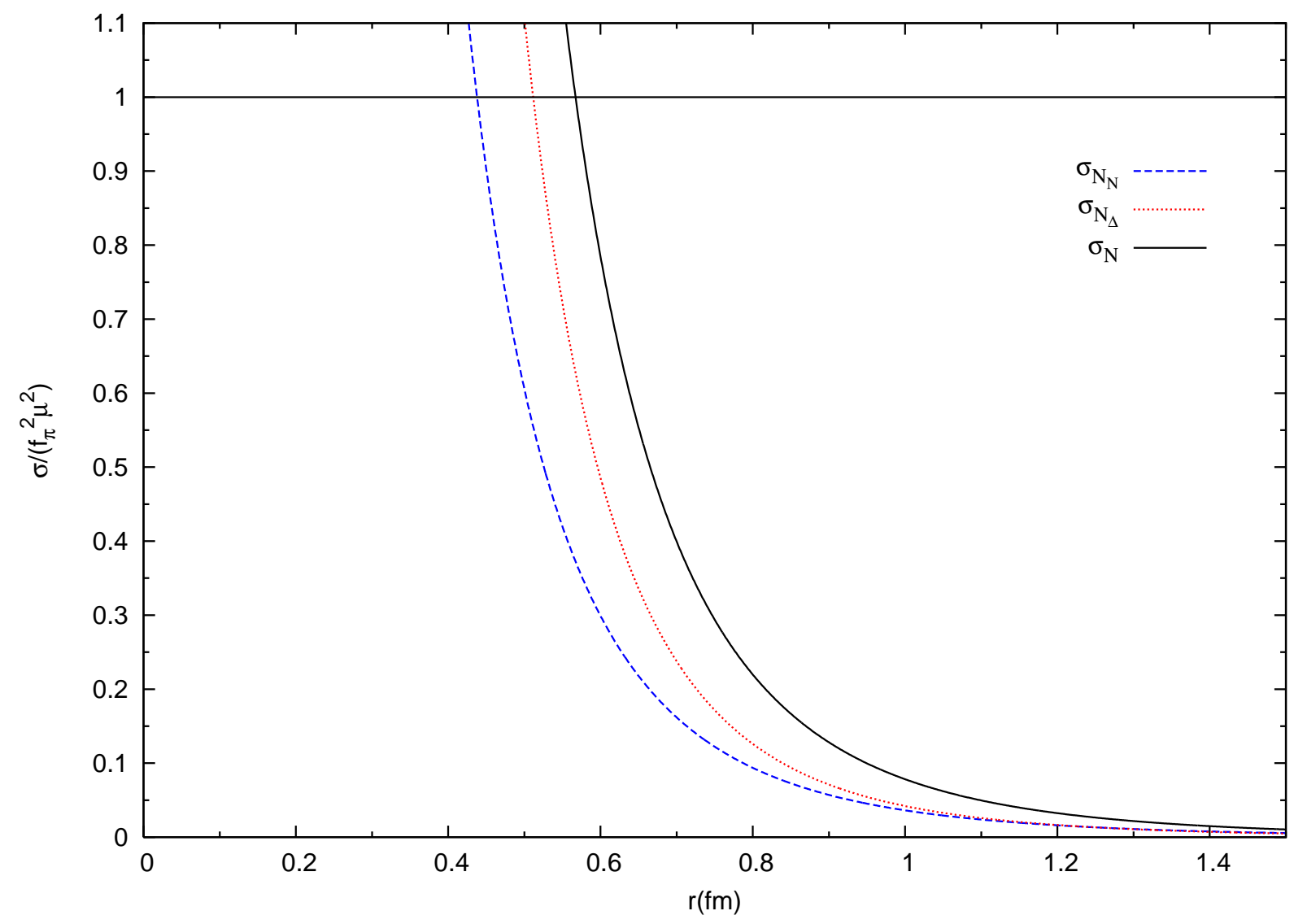

FIG. 2: Spatial dependence of the nucleon scalar form factor (continuous line) and partial contributions due to $N$ and $\Delta$ intermediate states [eq.(B4), dashed line and eq.(B5), dotted line, respectively].

Pions are Goldstone bosons, collective states derived from the $q \bar{q}$ condensate. The corre- 
sponding degrees of freedom are appropriately accomodated into non-linear lagrangians and described by the field $U=\exp (i \boldsymbol{\tau} \cdot \hat{\boldsymbol{\pi}} \theta)$, where $\hat{\boldsymbol{\pi}}$ is the isospin direction and $\theta$ is the chiral angle. This function can be expressed as $U=\cos \theta+i \boldsymbol{\tau} \cdot \hat{\boldsymbol{\pi}} \sin \theta$ and the dimensional pion field is given by

$$
\phi=f_{\pi} \sin \theta \hat{\pi}
$$

Long ago, Skyrme[11], in a series of papers, considered the possibility of pion fields being either weak or strong. It is worth noting that the words weak and strong, as used here, are akin to the notion of weak and strong electromagnetic fields developed by Schwinger, and not at all related to the nature of the fundamental interactions. In the former case, changes in the $q \bar{q}$ condensate are small, one relies on the approximation $\phi \simeq f_{\pi} \theta \hat{\boldsymbol{\pi}}$ and can employ perturbative techniques, as in chiral perturbation theory (ChPT). In the latter, disturbances of the QCD vacuum become important and the non-linear nature of pionic interactions manifests itself through the condition $|\phi| \leq f_{\pi}$. The physical picture behind eq.(6) is that pions, as Goldstone bosons, destroy the $q \bar{q}$ condensate in order to exist.

When strong fields are present, constraints also apply to the scalar form factor. The symmetry breaking lagrangian is written in terms of the dimensional pion field as

$$
\mathcal{L}_{s b}=\frac{1}{4} f_{\pi}^{2} \mu^{2} \operatorname{Tr}\left[U+U^{\dagger}\right]=f_{\pi}^{2} \mu^{2} \cos \theta .
$$

This structure shows that $\mathcal{L}_{s b}$ is a bound function and definition (2) means that the same necessarily happens with the scalar form factor. The function $\tilde{\sigma}_{N}(x)$ corresponds to a mass density induced in the vacuum by the presence of the nucleon, which manifests itself in the form of a pion cloud. Far away from the nucleon, eq.(7) yields the density of the condensate, which is negative and equal to $-f_{\pi}^{2} \mu^{2}$. In the description of a nucleon, it is convenient to use a convention for the energy in which the density tends to zero at long distances and $\mathcal{L}_{s b}$ is rewritten as

$$
\mathcal{L}_{s b}=f_{\pi}^{2} \mu^{2}(\cos \theta-1)
$$

In this new convention, the density vanishes when $r \rightarrow \infty$ and increases monotonically as one approaches the center of the nucleon as in fig.2. At a critical radius $R$ one has $\cos \theta=1$, the density becomes that of empty space and the condition

$$
\tilde{\sigma}_{N}(R)=f_{\pi}^{2} \mu^{2}
$$


holds. Beyond this point, a further increase in $\tilde{\sigma}_{N}$ would correspond to $\cos \theta>1$. In order to prevent this behaviour, we assume that the condensate no longer exists in the region $r<R$, and that the energy density saturates at $r=R$. For this reason, in our previous evaluation of $\sigma_{N}[9]$, we used the expression

$$
\sigma_{N}=\frac{4}{3} \pi R^{3} f_{\pi}^{2} \mu^{2}+4 \pi \int_{R}^{\infty} d r r^{2} \tilde{\sigma}_{N}(r)
$$

instead of eq.(4). This procedure is the basis of our model.

In the numerical determination of $\sigma_{N}$, we use the results of appendix B and consider two possibilities for the $\pi N \Delta$ coupling constant in the lagrangian (B2), corresponding to either the $S U(4)$ prediction $g_{\pi N \Delta}=3 g_{A} / 2 \sqrt{2}=1.33$ or $g_{\pi N \Delta}=1.47$, which yields $\Gamma=120 \mathrm{MeV}$ for the $\Delta$ decay width. The corresponding results, given in table I, are quite close to the value extracted from experiment by Gasser, Leutwyler and Sainio[6], namely $\sigma_{N}=45 \mathrm{MeV}$.

TABLE I: Nucleon $\sigma$-term as function of the $\pi N \Delta$ coupling constant.

\begin{tabular}{|c|c|c|}
\hline$g_{\pi N \Delta}$ & $R(\mathrm{fm})$ & $\sigma_{N}(\mathrm{MeV})$ \\
\hline \hline 1.33 & 0.57 & 45.8 \\
\hline 1.47 & 0.59 & 49.4 \\
\hline
\end{tabular}

Consistency with chiral symmetry is an important issue in this problem. Therefore, we note that, although the chiral powers of the pion mass expected from triangle diagrams are not explicit in the expressions of appendix B, the use of covariant relations among integrals[12] allows results for partial contributions to the $\sigma$-term to be recast in such a way that these powers become apparent, as shown in appendix C. In appendix D we show that the formal chiral expansion of eq.(10) gives rise to the expected non-analytic terms (log $\mu$ and $\mu^{3}$ ) and agrees fully with that produced by standard chiral perturbation theory[8], provided the renormalization scale is identified with $1 / R$.

\section{DELTA $\sigma$-TERM}

The delta scalar form factor is defined as

$$
<\Delta\left(p^{\prime}, s^{\prime}\right)\left|-\mathcal{L}_{s b}\right| \Delta(p, s)>\equiv-\bar{u}_{\mu}^{s^{\prime}}\left(\boldsymbol{p}^{\prime}\right)\left[g^{\mu \nu} \sigma_{\Delta}(t)+p^{\prime \nu} p^{\mu} F_{T}(t)\right] u_{\nu}^{s}(\boldsymbol{p})
$$


where $u_{\nu}^{s}$ is the $\Delta$ spinor[13] and $\sigma_{\Delta}$ and $F_{T}$ are respectively the scalar and tensor form factors. The minus sign on the r.h.s. is associated with the conventions used in the free $\Delta$ lagrangian as in ref.[1]. We assume that the scalar form factor is determined by a short range contact interaction and the two long range two-pion processes shown in fig.1.

In figs. 3 and 4 (zoom in) we display the profile functions for the partial contributions to $\sigma_{\Delta}$ given by eqs.(B9,B10) and it is interesting to note that the nucleon contribution oscillates in the outer region, in sharp contrast with fig.2. This behaviour is due to the fact that the delta is unstable and makes $\sigma_{\Delta}$ to be smaller than $\sigma_{N}$. The structure of partial contributions for $S U(4)$ coupling constants is given in table II, where core and cloud refer respectively to regions inside and outside the cutting radius $R$.

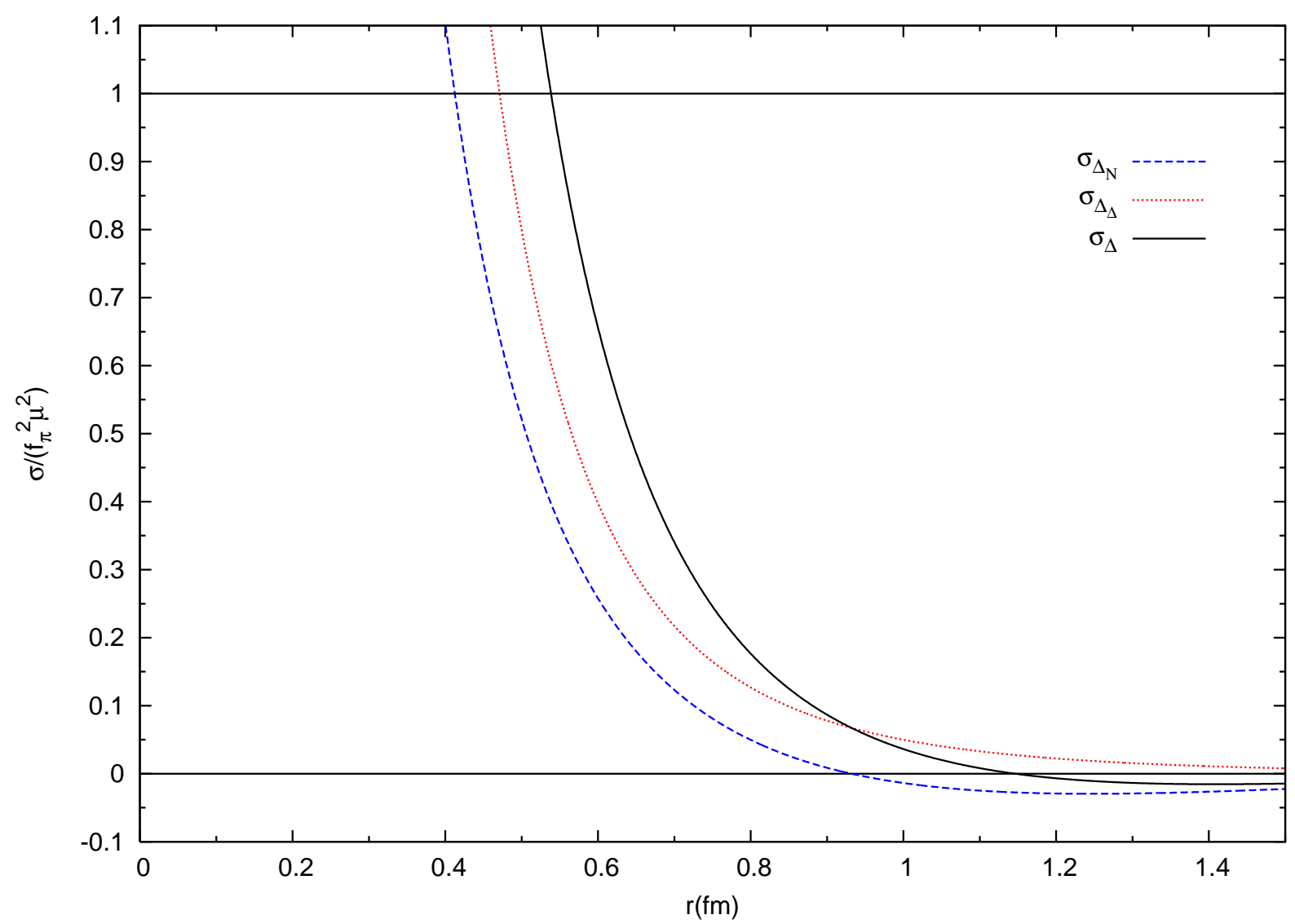

FIG. 3: Spatial dependence of the delta scalar form factor (continuous line) and partial contributions due to $N$ and $\Delta$ intermediate states [eq.(B9), dashed line and eq.(B10), dotted line, respectively]. 


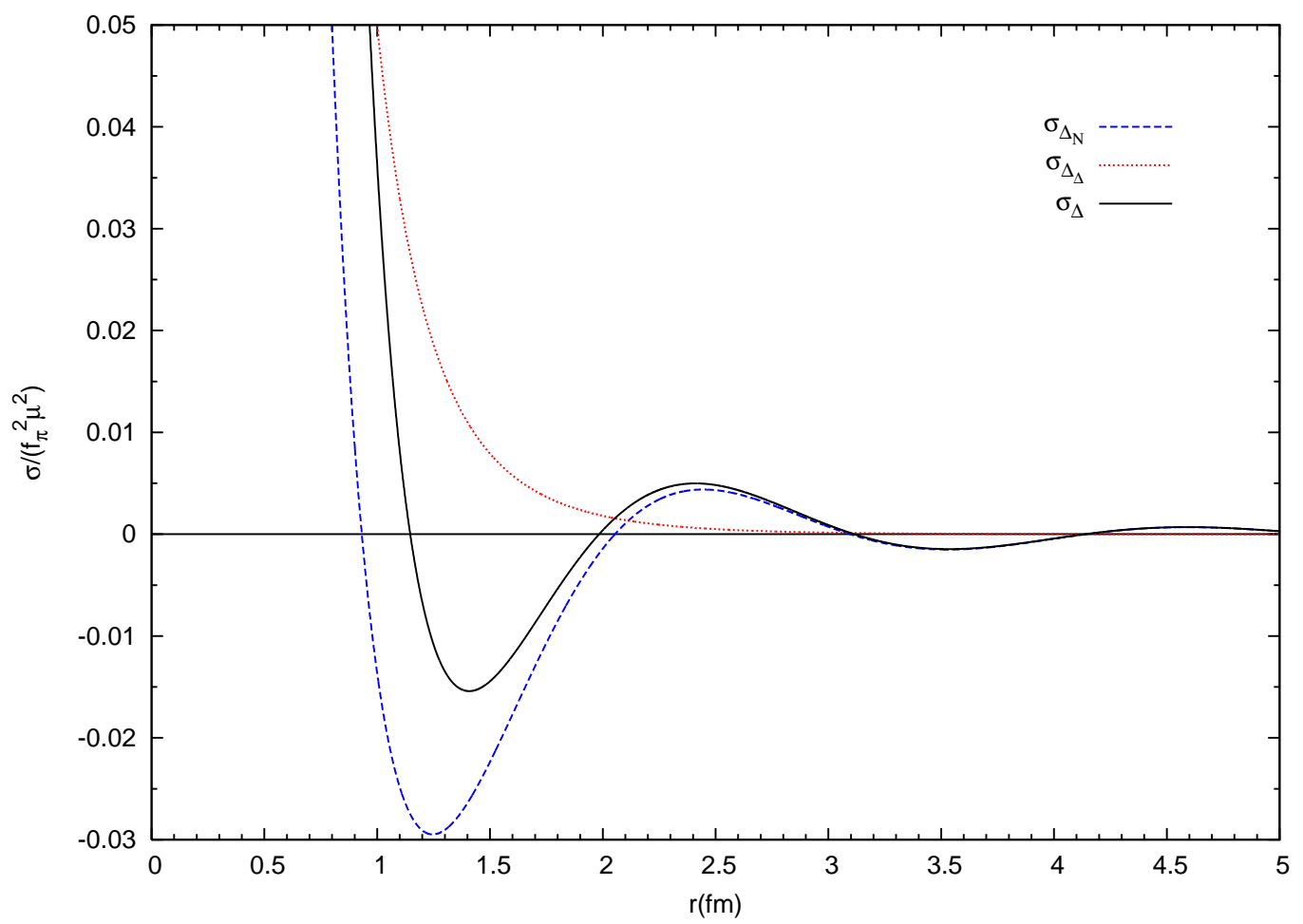

FIG. 4: Expanded portion of fig.3, with the same conventions.

TABLE II: Partial contributions to $\sigma_{N}$ [eqs.(B4,B5)] and $\sigma_{\Delta}$ [eqs.(B9,B10)].

\begin{tabular}{|c|c|c|c|c|}
\hline & core & cloud $N$ & cloud $\Delta$ & sum \\
\hline \hline$\sigma_{N}(\mathrm{MeV})$ & 16.7 & 13.0 & 16.1 & 45.8 \\
\hline$\sigma_{\Delta}(\mathrm{MeV})$ & 14.3 & -1.5 & 19.3 & 32.1 \\
\hline
\end{tabular}

Processes containing nucleon intermediate states give rise to an imaginary component $\sigma_{\Delta}^{I}$ for the delta $\sigma$-term, which can be related to the decay width by means of the FeynmanHellmann theorem[5]:

$$
\sigma_{\Delta}-i \sigma_{\Delta}^{I}=\mu^{2} \frac{d(M-i \Gamma / 2)}{d \mu^{2}}
$$


Using $[8]$

$$
\begin{aligned}
& \Gamma=\frac{g_{\pi N \Delta}^{2}}{24 \pi M^{2} f_{\pi}^{2}} q_{\Delta}^{3}\left[(M+m)^{2}-\mu^{2}\right] \\
& q_{\Delta}=\frac{1}{2 M} \sqrt{M^{4}+m^{4}+\mu^{4}-2 m^{2} M^{2}-2 \mu^{2} M^{2}-2 \mu^{2} m^{2}}
\end{aligned}
$$

one finds

$$
\sigma_{\Delta}^{I}=-\frac{g_{\pi N \Delta}^{2} \mu^{2}}{48 \pi M^{2} f_{\pi}^{2}}\left\{q_{\Delta}^{3}+3 q_{\Delta} \frac{M^{2}+m^{2}-\mu^{2}}{4 M^{2}}\left[(M+m)^{2}-\mu^{2}\right]\right\} .
$$

The values of the distance $R$ for which $\tilde{\sigma}_{\Delta}(R) / f_{\pi}^{2} \mu^{2}=1$ and of the delta $\sigma$-term, calculated by means of eqs.(B9, B10), are given in table III, for different choices of the coupling constants $g_{\pi N \Delta}$ and $g_{\pi \Delta \Delta}$. The $S U(4)$ predictions for these constants are 1.33 and 0.75 , whereas the value 1.47 for the former yields the empirical decay width. The value 0.67 for the latter was used in ref.[3]. Results for the real component of $\sigma_{\Delta}$ are sensitive to the coupling constant $g_{\pi \Delta \Delta}$ and fully consistent with that given in ref.[4], namely $\sigma_{\Delta}=(32 \pm 3)$ $\mathrm{MeV}$. On the other hand, our prediction is larger than that quoted in ref.[3]. The values for the imaginary component $\sigma_{\Delta}^{I}$, obtained by means of eq.(B9), are identical with those given by eq.(14), as they should.

TABLE III: Real and imaginary parts of the $\Delta \sigma$-term as function of the coupling constants.

\begin{tabular}{|c|c|c|c|c|}
\hline$g_{\pi N \Delta}$ & $g_{\pi \Delta \Delta}$ & $R(\mathrm{fm})$ & $\operatorname{Re} \sigma_{\Delta}(\mathrm{MeV})$ & $\operatorname{Im} \sigma_{\Delta}(\mathrm{MeV})$ \\
\hline \hline 1.33 & 0.75 & 0.54 & 32.1 & -21.7 \\
\hline 1.33 & 0.67 & 0.52 & 28.1 & -21.7 \\
\hline 1.47 & 0.75 & 0.55 & 31.7 & -26.7 \\
\hline 1.47 & 0.67 & 0.53 & 27.8 & -26.7 \\
\hline
\end{tabular}

\section{SUMMARY}

We have discussed a model aimed at determining $\sigma$-terms, which consists in cutting off configuration space expressions at the point where the cosine of the chiral angle becomes larger than 1. The model has been used to calculate $\sigma_{N}$ and $\sigma_{\Delta}$ with success. In the 
former case, a value very close to that extracted from experiment by Gasser, Leutwyler and Sainio[6], was obtained. In the case of the delta, the prediction $28 \mathrm{MeV} \leq \sigma_{\Delta} \leq 32 \mathrm{MeV}$, depending on the coupling constants employed, is also very close to the result produced by another group[4]. The fact that the delta can decay gives rise to a pion cloud which includes an oscillation and is responsible for both the relation $\sigma_{\Delta}<\sigma_{N}$ and the consistency of the imaginary part of $\sigma_{\Delta}$ with the decay width. Analytic expressions also comply with chiral counting rules and give rise to expected non-analytic terms in the chiral limit. These features suggest that our calculational procedure is sound and can be reliably applied to other systems.

\section{APPENDIX A: LOOP INTEGRALS}

In the triangle diagrams, $p$ and $p^{\prime}$ are the initial and final baryon momenta, whereas $k$ and $k^{\prime}$ are the momenta of the exchanged pions. We also employ the variables

$$
q=\left(p-p^{\prime}\right), \quad P=\left(p+p^{\prime}\right) / 2, \quad Q=\left(k+k^{\prime}\right) / 2 .
$$

In all diagrams, the external baryon, with mass $m_{e}$, is assumed to be on shell and one has

$$
\begin{aligned}
p^{2}=p^{\prime 2}=m_{e}^{2}, & P \cdot q=0, \\
\bar{u} \not q u=0, & \bar{u} P u=m \bar{u} u, \\
\bar{u}^{\mu} \not q u^{\nu}=0, & \bar{u}^{\mu} P u^{\nu}=M \bar{u}^{\mu} u^{\nu} .
\end{aligned}
$$

The basic loop integrals needed in this work involve either two or three denominators. We use the definition

$$
\int[\cdots]=\int \frac{d^{4} Q}{(2 \pi)^{4}} \frac{1}{\left[(Q+q / 2)^{2}-\mu^{2}\right]\left[(Q-q / 2)^{2}-\mu^{2}\right]}
$$


and the dimensionless expressions

$$
\begin{aligned}
I_{\pi \pi} & =\int[\cdots]=\frac{i}{(4 \pi)^{2}} \Pi_{\pi \pi}^{(00)}, \\
I_{\pi \pi}^{\mu \nu} & =\int[\cdots] \frac{Q^{\mu} Q^{\nu}}{\mu^{2}}=\frac{i}{(4 \pi)^{2}}\left[g^{\mu \nu} \bar{\Pi}_{\pi \pi}^{(00)}+\cdots\right] \\
I_{x \pi \pi} & =\int[\cdots] \frac{2 \mu m_{e}}{\left[(Q+P)^{2}-m_{x}^{2}\right]}=\frac{i}{(4 \pi)^{2}} \Pi_{x \pi \pi}^{(000)}, \\
I_{x \pi \pi}^{\mu} & =\int[\cdots] \frac{\left(Q^{\mu} / \mu\right) 2 \mu m_{e}}{\left[(Q+P)^{2}-m_{x}^{2}\right]}=\frac{i}{(4 \pi)^{2}}\left[\frac{P^{\mu}}{m_{e}} \Pi_{x \pi \pi}^{(100)}+\cdots\right], \\
I_{x \pi \pi}^{\mu \nu} & =\int[\cdots] \frac{\left(Q^{\mu} Q^{\nu} / \mu^{2}\right) 2 \mu m_{e}}{\left[(Q+P)^{2}-m_{x}^{2}\right]}=\frac{i}{(4 \pi)^{2}}\left[g^{\mu \nu} \bar{\Pi}_{x \pi \pi}^{(000)}+\cdots\right], \\
I_{x \pi \pi}^{\mu \nu \rho} & =\int[\cdots] \frac{\left(Q^{\mu} Q^{\nu} Q^{\rho} / \mu^{3}\right) 2 \mu m_{e}}{\left[(Q+P)^{2}-m_{x}^{2}\right]}=\frac{i}{(4 \pi)^{2}}\left[g^{\mu \nu} \frac{P^{\rho}}{m_{e}} \bar{\Pi}_{x \pi \pi}^{(100)}+\cdots\right] .
\end{aligned}
$$

where the ellipses indicate terms that do not contribute to the scalar form factors. The usual Feynman techniques for loop integration allow one to write the regular parts of these integrals as

$$
\begin{aligned}
& \Pi_{\pi \pi}^{(00)}=-\int_{0}^{1} d a \ln \left(\frac{D_{\pi \pi}}{\mu^{2}}\right), \\
& \bar{\Pi}_{\pi \pi}^{(00)}=-\int_{0}^{1} d a \frac{D_{\pi \pi}}{2 \mu^{2}} \ln \left(\frac{D_{\pi \pi}}{\mu^{2}}\right), \\
& \Pi_{x \pi \pi}^{(k 00)}=-\int_{0}^{1} d a a \int_{0}^{1} d b\left[-m_{e}(1-a) / \mu\right]^{k}\left(\frac{2 \mu m_{e}}{D_{x \pi \pi}}\right), \\
& \bar{\Pi}_{x \pi \pi}^{(k 00)}=-\frac{m_{e}}{\mu} \int_{0}^{1} d a a \int_{0}^{1} d b\left[-m_{e}(1-a) / \mu\right]^{k} \ln \left(\frac{D_{x \pi \pi}}{2 \mu m_{e}}\right),
\end{aligned}
$$

with

$$
\begin{aligned}
& D_{\pi \pi}=\mu^{2}-a(1-a) q^{2} \\
& D_{x \pi \pi}=a \mu^{2}+(1-a) m_{x}^{2}-a(1-a) m_{e}^{2}-a^{2} b(1-b) q^{2} .
\end{aligned}
$$

The dimensionless configuration space functions $S$ are defined as

$$
S=\int \frac{d \boldsymbol{k}}{(2 \pi)^{3}} e^{-i \boldsymbol{k} \cdot \boldsymbol{x}} \Pi
$$


with $x=\mu r$ and $\boldsymbol{k}=\boldsymbol{q} / \mu$. Performing the Fourier transforms, we find

$$
\begin{aligned}
S_{\pi \pi}^{(00)} & =\frac{1}{\pi x^{2}} K_{1}(2 x), \\
\bar{S}_{\pi \pi}^{(00)} & =-\frac{1}{2 \pi x^{4}}\left[x K_{0}(2 x)+K_{1}(2 x)\right], \\
\phi^{2}>0 \rightarrow S_{x \pi \pi}^{(k 00)} & =-\frac{2 m_{e}}{\mu} \frac{1}{\pi x} \int_{0}^{1} d a \frac{\left[-m_{e}(1-a) / \mu\right]^{k}}{a} K_{0}(2 \phi x), \\
\phi^{2}<0 \rightarrow S_{x \pi \pi}^{(k 00)} & =\frac{m_{e}}{\mu} \frac{1}{x} \int_{0}^{1} d a \frac{\left[-m_{e}(1-a) / \mu\right]^{k}}{a}\left[Y_{0}(2|\phi| x)+i J_{0}(2|\phi| x)\right], \\
\phi^{2}>0 \rightarrow \bar{S}_{x \pi \pi}^{(k 00)} & =\frac{1}{\pi x^{2}} \frac{m_{e}}{\mu} \int_{0}^{1} d a a\left[-m_{e}(1-a) / \mu\right]^{k} \phi K_{1}(2 \phi x), \\
\phi^{2}<0 \rightarrow \bar{S}_{x \pi \pi}^{(k 00)} & =-\frac{1}{2 x^{2}} \frac{m_{e}}{\mu} \int_{0}^{1} d a a\left[-m_{e}(1-a) / \mu\right]^{k}|\phi|\left[Y_{1}(2|\phi| x)+i J_{1}(2|\phi| x)\right],
\end{aligned}
$$

with

$$
\phi^{2}=\left[a \mu^{2}+(1-a) m_{x}^{2}-a(1-a) m_{e}^{2}\right] /\left(\mu^{2} a^{2}\right)
$$

\section{APPENDIX B: SCALAR FORM FACTORS}

We give here the expressions for $\tilde{\sigma}_{B_{I}}$, due to triangle diagrams containing external states $B$ and an intermediate states $I$. The following interaction lagrangians $[1,8,13,14]$ are used

$$
\begin{aligned}
\mathcal{L}_{\pi N N} & =\frac{g_{A}}{2 f_{\pi}}\left\{\bar{N} \gamma_{\mu} \gamma_{5} \tau_{a} N\right\} \cdot \partial^{\mu} \phi_{a}, \\
\mathcal{L}_{\pi N \Delta} & =\frac{g_{\pi N \Delta}}{f_{\pi}}\left\{\bar{\Delta}^{\mu}\left[g_{\mu \nu}-(Z-1 / 2) \gamma_{\mu} \gamma_{\nu}\right] M_{a} N\right\} \cdot \partial^{\nu} \phi_{a}+\text { h.c. }, \\
\mathcal{L}_{\pi \Delta \Delta} & =-\frac{g_{\pi \Delta \Delta}}{f_{\pi}}\left\{\bar{\Delta}^{\mu}\left(g_{\mu \nu} \gamma_{\lambda}-g_{\mu \lambda} \gamma_{\nu}-g_{\lambda \nu} \gamma_{\mu}\right) \gamma_{5} T_{a} \Delta^{\nu}\right\} \cdot \partial^{\lambda} \phi_{a},
\end{aligned}
$$

where $\phi, N$ and $\Delta$ denote pion, nucleon and delta fields, $f_{\pi}$ is the pion decay constant, $g_{A}$, $g_{\pi N \Delta}$ and $g_{\pi \Delta \Delta}$ are coupling constants, and $\tau, M$ and $T$ are matrices that couple nucleons and deltas into isospin 1 states, with $\tau_{a} \tau_{a}=3, M_{a}^{\dagger} M_{a}=2, M_{a} M_{a}^{\dagger}=1$, and $T_{a}^{\dagger} T_{a}=15 / 4$. For the coupling constants we use $g_{A}=1.25$ and the $S U(4)$ results $g_{\pi N \Delta}=3 g_{A} / 2 \sqrt{2}$ and $g_{\pi \Delta \Delta}=3 g_{A} / 5$. We also use $f_{\pi}=93 \mathrm{MeV}, \mu=139.57 \mathrm{MeV}, m=938.27 \mathrm{MeV}$ and $M=1232 \mathrm{MeV}$. 


\section{nucleon:}

Using the loop integrals $S$ defined in appendix A, we obtain the following contributions to the nucleon scalar form factor

$$
\begin{aligned}
& \tilde{\sigma}_{N_{N}}(r)=\frac{3}{4}\left[\frac{\mu g_{A}}{4 \pi f_{\pi}}\right]^{2} 2 m\left(\mu^{3}\right)\left\{S_{\pi \pi}^{(00)}-S_{N \pi \pi}^{(100)}\right\} \\
& \tilde{\sigma}_{N_{\Delta}}(r)=2\left[\frac{\mu g_{\pi N \Delta}}{4 \pi f_{\pi}}\right]^{2} \frac{(m+M)}{6 M^{2}}\left(\mu^{3}\right)\left\{-\left[(m+M)(2 M-m)+2 \mu^{2}+\frac{m \mu^{2}\left(1-\nabla^{2} / 2\right)}{(m+M)}\right] S_{\pi \pi}^{(00)}\right. \\
& -\frac{2 m \mu^{2}}{(m+M)} \bar{S}_{\pi \pi}^{(00)} \\
& +\frac{1}{2 m \mu}\left[\left(m^{2}-M^{2}\right)(m+M)(2 M-m)+2 \mu^{2}\left(m^{2}-M^{2}\right)+6 M^{2} \mu^{2}\left(1-\nabla^{2} / 2\right)\right] S_{\Delta \pi \pi}^{(000)} \\
& \left.+\frac{1}{2 m}\left[(m+M)\left(4 m M-M^{2}-m^{2}\right)-2 \mu^{2}(2 M-m)+\frac{6 M^{2} \mu^{2}}{(m+M)}\left(1-\nabla^{2} / 2\right)\right] S_{\Delta \pi \pi}^{(100)}\right\} .
\end{aligned}
$$

\section{delta:}

In the evaluation of the triangle diagram, the external deltas are on shell and one has the constraints $p \cdot u^{s}(\boldsymbol{p})=\gamma \cdot u^{s}(\boldsymbol{p})=\bar{u}^{s^{\prime}}\left(\boldsymbol{p}^{\prime}\right) \cdot p^{\prime}=\bar{u}^{s^{\prime}}\left(\boldsymbol{p}^{\prime}\right) \cdot \gamma=0$. The $T$ matrix can be cast in the form

$$
i T=\bar{u}_{\mu}^{s^{\prime}}\left(\boldsymbol{p}^{\prime}\right)\left\{\int \frac{d^{4} Q}{(2 \pi)^{4}} \frac{\Theta^{\mu \nu}}{\left[(Q-q / 2)^{2}-\mu^{2}\right]\left[(Q+q / 2)^{2}-\mu^{2}\right]}\right\} u_{\nu}^{s}(\boldsymbol{p})
$$

with

$$
\begin{aligned}
& \Theta_{N}^{\mu \nu}=2\left[\frac{\mu g_{\pi N \Delta}}{f_{\pi}}\right]^{2}(Q+q / 2)^{\mu}(Q-q / 2)^{\nu} \frac{m+M+\varnothing}{\bar{p}^{2}-m^{2}} \\
& \Theta_{\Delta}^{\mu \nu}=\frac{15}{4}\left[\frac{\mu g_{\pi \Delta \Delta}}{f_{\pi}}\right]^{2}\left[g^{\mu \nu}\left(2 M-\frac{4 M^{2}}{\bar{p}^{2}-M^{2}}\right) \not-\frac{8}{3}(Q+q / 2)^{\mu}(Q-q / 2)^{\nu} \frac{M-\not}{\bar{p}^{2}-m^{2}}\right]
\end{aligned}
$$

with $\bar{p}=P+Q$. Performing the integrals, comparing the results with eq.(2), and going to configuration space, we obtain the contributions

$$
\begin{aligned}
& \tilde{\sigma}_{\Delta_{N}}(r)=\left[\frac{\mu g_{\pi N \Delta}}{4 \pi f_{\pi}}\right]^{2} \mu\left(\mu^{3}\right)\left[\frac{(m+M)}{2 M} \bar{S}_{N \pi \pi}^{(000)}+\frac{\mu}{2 M} \bar{S}_{N \pi \pi}^{(100)}\right] \\
& \tilde{\sigma}_{\Delta_{\Delta}}(r)=\frac{15}{4}\left[\frac{\mu g_{\pi \Delta \Delta}}{4 \pi f_{\pi}}\right]^{2} 2 M\left(\mu^{3}\right)\left[S_{\pi \pi}^{(00)}-S_{\Delta \pi \pi}^{(100)}-\frac{2 \mu}{3 M} \bar{S}_{\Delta \pi \pi}^{(000)}+\frac{2 \mu^{2}}{3 M^{2}} \bar{S}_{\Delta \pi \pi}^{(100)}\right] .
\end{aligned}
$$

\section{APPENDIX C: CHIRAL SYMMETRY}

In this appendix we show that results $(\mathrm{B} 4, \mathrm{~B} 5)$ and $(\mathrm{B} 9, \mathrm{~B} 10)$ are fully compatible with standard chiral power counting by means of a covariant chiral expansion[12]. It is important 
to note that these expressions contain a factor $\left(\mu^{3}\right)$, which comes from the definition of the configuration space function $S$ and must not be included in the counting. With this previous in mind, we use the following relations among integrals,

$$
\begin{gathered}
S_{\pi \pi}^{(00)}=\left[1-\frac{\mu^{2} \nabla^{2}}{4 m_{e}^{2}}\right] S_{x \pi \pi}^{(100)}+\left[\frac{\mu}{2 m_{e}}\left(1-\nabla^{2} / 2\right)+\frac{\left(m_{e}^{2}-m_{x}^{2}\right)}{2 \mu m_{e}}\right] S_{x \pi \pi}^{(000)} \\
2\left[1-\frac{\mu^{2} \nabla^{2}}{4 m_{e}^{2}}\right] \bar{S}_{x \pi \pi}^{(000)}=\left[\frac{\mu}{2 m_{e}}\left(1-\nabla^{2} / 2\right)+\frac{\left(m_{e}^{2}-m_{x}^{2}\right)}{2 \mu m_{e}}\right] S_{\pi \pi}^{(00)} \\
-\left[\frac{\left(m_{e}^{2}-m_{x}^{2}\right)^{2}}{4 \mu^{2} m_{e}^{2}}-\frac{m_{e}^{2}+m_{x}^{2}}{2 m_{e}^{2}}+\frac{\mu^{2}}{4 m_{e}^{2}}+\frac{m_{x}^{2}}{4 m_{e}^{2}} \nabla^{2}\right] S_{x \pi \pi}^{(000)} \\
2\left[1-\frac{\mu^{2} \nabla^{2}}{4 m_{e}^{2}}\right] \bar{S}_{x \pi \pi}^{(100)}=-\frac{1}{3}\left(1-\nabla^{2} / 4\right) S_{\pi \pi}^{(00)} \\
-\left[\frac{\left(m_{e}^{2}-m_{x}^{2}\right)^{2}}{4 \mu^{2} m_{e}^{2}}-\frac{m_{e}^{2}+m_{x}^{2}}{2 m_{e}^{2}}+\frac{\mu^{2}}{4 m_{e}^{2}}+\frac{m_{x}^{2}}{4 m_{e}^{2}} \nabla^{2}\right] S_{x \pi \pi}^{(100)}
\end{gathered}
$$

which are obtained by multiplying eqs.(A8-A10) by $P_{\mu}$, neglecting short range terms with a single pion propagator, and going to configuration space. In the case $m_{e}=M$ and $m_{x}=m$, the expansion of eqs.(A8-A9) yield the leading order relations

$$
\begin{aligned}
\Pi_{\Delta \pi \pi}^{(000)} & \simeq \frac{\left(2 m_{e} \mu\right)}{\left(m^{2}-M^{2}\right)} \Pi_{\pi \pi}^{(00)}, \\
\Pi_{\Delta \pi \pi}^{(100)} & \simeq-\frac{\left(2 m_{e} \mu\right)^{2}}{\left(m^{2}-M^{2}\right)^{2}} \bar{\Pi}_{\pi \pi}^{(00)} .
\end{aligned}
$$

Truncating the expansions at $\mathcal{O}\left(q^{4}\right)$, we find

$$
\begin{aligned}
& \tilde{\sigma}_{N_{N}}(r)=\frac{3}{4}\left[\frac{\mu g_{A}}{4 \pi f_{\pi}}\right]^{2} \mu\left(\mu^{3}\right)\left[\left(1-\nabla^{2} / 2\right) S_{N \pi \pi}^{(000)}-\frac{\mu}{2 m} \nabla^{2} S_{\pi \pi}^{(00)}\right] \\
& \tilde{\sigma}_{N_{\Delta}}(r)=-\frac{4}{3}\left[\frac{\mu g_{\pi N \Delta}}{4 \pi f_{\pi}}\right]^{2} \frac{\mu^{2}}{(M-m)}\left(\mu^{3}\right)\left[\left(1-\nabla^{2} / 2\right)-\frac{m^{2}}{3 M^{2}}\left(1-\nabla^{2} / 4\right)\right] S_{\pi \pi}^{(00)}, \\
& \tilde{\sigma}_{\Delta_{N}}(r)=\frac{1}{4}\left[\frac{\mu g_{\pi N \Delta}}{4 \pi f_{\pi}}\right]^{2} \frac{(M+m)}{M} \mu\left(\mu^{3}\right)\left[\frac{(M+m)}{4 \mu M^{2}}\left(M^{2}-m^{2}\right) S_{N \pi \pi}^{(100)}\right. \\
& \left.+\left(1-\frac{M-m}{6 M}\right)\left(1-\frac{\nabla^{2}}{4}\right) S_{N \pi \pi}^{(000)}\right], \\
& \tilde{\sigma}_{\Delta_{\Delta}}(r)=\frac{15}{4}\left[\frac{\mu g_{\pi \Delta \Delta}}{4 \pi f_{\pi}}\right]^{2} \mu\left(\mu^{3}\right)\left[\left(1-\nabla^{2} / 2\right) S_{\Delta \pi \pi}^{(000)}-\frac{\mu \nabla^{2}}{2 M} S_{\Delta \pi \pi}^{(100)}\right. \\
& \left.-\frac{4}{3} \bar{S}_{\Delta \pi \pi}^{(000)}+\frac{4 \mu}{3 M} \bar{S}_{\Delta \pi \pi}^{(100)}\right] .
\end{aligned}
$$


These results, except for eq. C8, which contains imaginary terms, are compatible with chiral counting rules. Contributions begin at $\mathcal{O}\left(q^{3}\right)$ for diagrams in which internal and external baryons are identical and at $\mathcal{O}\left(q^{4}\right)$ when this does not happen.

\section{APPENDIX D: CHIRAL LIMIT}

In this section we show that our model for the nucleon $\sigma$-term is consistent with the standard ChPT expansion. In the paper by Becher and Leutwyler[8], one finds, using our notation

$$
\begin{aligned}
& \sigma_{N}=-4 c_{1} \mu^{2}-\frac{9 g_{A}^{2} \mu^{3}}{64 \pi f_{\pi}^{2}}-\frac{3 \mu^{4}}{16 \pi^{2} f_{\pi}^{2} m}\left(g_{A}^{2}-8 c_{1} m+c_{2} m+4 c_{3} m\right) \ln \frac{\mu}{m} \\
& -\frac{3 \mu^{4}}{64 \pi^{2} f_{\pi}^{2} m}\left(3 g_{A}^{2}-8 c_{1} m+4 c_{3} m\right)+2 \bar{e}_{1},
\end{aligned}
$$

where $c_{i}$ and $e_{1}$ are, respectively, low enegy constants (LECs) from the $\mathcal{L}_{N}^{(2)}$ and $\mathcal{L}_{N}^{(4)}$ lagrangians. The bar over $e_{1}$ indicates that it has been renormalized.

In order to expand our $\sigma_{N}$, we use in eq.(C4) the result

$$
S_{N \pi \pi}^{(000)} \simeq-\frac{e^{-2 x}}{2 x^{2}}+\frac{\mu}{m \pi x^{2}}\left[x K_{0}(2 x)+K_{1}(2 x)\right]
$$

which holds[15] for $\mu / m<<1$. This allows integrations in eq.(10) to be performed analytically and one finds

$$
\begin{aligned}
& \sigma_{N}=\frac{4}{3} \pi R^{3} f_{\pi}^{2} \mu^{2}+\frac{3 g_{A}^{2} \mu^{3}}{16 \pi f_{\pi}^{2}}\left\{\left(\frac{1}{4}+\frac{1}{2 \mu R}\right) e^{-2 \mu R}-\frac{\mu}{2 m \pi}\left[4 K_{0}(2 \mu R)\right.\right. \\
& \left.\left.+\left(2 \mu R+\frac{6}{2 \mu R}\right) K_{1}(2 \mu R)\right]\right\}+\frac{g_{\pi N \Delta}^{2} \mu^{4}}{6 \pi^{2} f_{\pi}^{2}(M-m)}\left[K_{0}(2 \mu R)+\left(3-\frac{m^{2}}{2 M^{2}}\right) \frac{K_{1}(2 \mu R)}{2 \mu R}\right] .
\end{aligned}
$$

An expansion for small values of $\mu$ yields

$$
\begin{aligned}
& \sigma_{N}=\frac{4}{3} \pi R^{3} f_{\pi}^{2} \mu^{2} \\
& +\frac{3 g_{A}^{2}}{16 \pi f_{\pi}^{2}}\left[\frac{\mu^{2}}{2 R^{2}}\left(R-\frac{1}{m \pi}\right)-\frac{3 \mu^{3}}{4}-\frac{\mu^{4}}{m \pi} \ln \mu R+\frac{\mu^{4}(5-4 \gamma)}{4 m \pi}+\frac{\mu^{4} R}{2}\right] \\
& +\frac{g_{\pi N \Delta}^{2}}{12 \pi^{2} f_{\pi}^{2}(M-m)}\left[\frac{\mu^{2}}{R^{2}}\left(3-\frac{m^{2}}{2 M^{2}}\right)+\mu^{4}\left(4-\frac{m^{2}}{M^{2}}\right) \ln \mu R+\mu^{4}\left(-3+4 \gamma+\frac{m^{2}}{2 M^{2}}(1-2 \gamma)\right)\right],
\end{aligned}
$$

where $\gamma$ is the Euler constant. This result reproduces the first three terms of eq.(D1), provided one absorbs the factors proportional to $\mu^{2}$ into the definition of $c_{1}$, uses the delta 
contributions to the $c_{i}$, which are given by

$$
c_{1}^{\Delta}=0, \quad c_{2}^{\Delta}=\frac{4 g_{\pi N \Delta}^{2} m^{2}}{9 M^{2}(M-m)}, \quad c_{3}^{\Delta}=\frac{-4 g_{\pi N \Delta}^{2}}{9(M-m)},
$$

and chooses the value $R=1 / m$ for the cutting radius. As the renormalized constant $\bar{e}_{1}$ contains factors proportional to $\mu^{4}$, terms of this kind need not to coincide.

\section{ACKNOWLEDGMENTS}

The works by I.P.C. and D.O.S. were supported by CNPq and the work by G.R.S.Z., by FAPESP (Brazilian agencies).

[1] T. R. Hemmert, B. R. Holstein and J. Kambor, J. Phys. G 24, 1831 (1998).

[2] N. Kaiser, S. Gerstendörfer and W. Weise, Nucl. Phys. A 637, 395 (1998); N. Fettes and G-Ulf. Meissner, Nucl. Phys. A 679, 629 (2001).

[3] V. Bernard, T. R. Hemmert and Ulf-G. Meissner, preprint hep-lat/0503022.

[4] V. E. Lyubovitskij, Th. Gutsche, A. Faessler and E. G. Drukarev, Phys. Rev. D 63, 054026 (2001).

[5] H. Hellmann, Einführung in die Quantenchemie (Deuticke Verlag, Leipzig, 1937); R.P. Feynman, Phys. Rev. 56, 340 (1939); S. T. Epstein, Am. J. Phys. 22, 613 (1954); J. Gasser, M. E. Sainio and A. Švarc, Nucl. Phys. B307, 779 (1988).

[6] J. Gasser, H. Leutwyler and M.E. Sainio, Phys. Lett. B 253, 252 (1991); 253, 260 (1991).

[7] J. Gasser, M.E. Sainio and A. Švarc, Nucl. Phys. B 307, 779 (1988).

[8] T. Becher and H. Leutwyler, Eur. Phys. Journal C 9, 643 (1999); JHEP 106, 17 (2001).

[9] M.R. Robilotta, Phys. Rev. C 63, 044004 (2001).

[10] A.I. L'vov, S. Scherer, B. Pasquini, C. Unkmeir and D. Drechsel, Phys. Rev. C 64, 015203 (2001).

[11] T. H. R. Skyrme, Proc. Roy. Soc. London A247, 260 (1958); A252, 236 (1959); A260, 127 (1961); Nucl. Phys. 31, 556 (1962).

[12] R. Higa and M. R. Robilotta, Phys. Rev. C 68, 024004 (2003).

[13] C. Fronsdal, N. Cim. Suppl. 9, 416 (1958). 
[14] G. Höhler, group I, vol.9, subvol.b, part 2 of Landölt-Bornstein Numerical data and Functional Relationships in Science and Technology, ed. H.Schopper (1983).

[15] R. Higa, M. R. Robilotta and C. A da Rocha, Phys. Rev. C 69, 034009 (2004). 